\title{
Enhancing Mass Transport in Redox Flow Batteries by Tailoring Flow Field and Electrode Design
}

\author{
C. R. Dennison, ${ }^{\text {a,b }}$ Ertan Agar, ${ }^{c, *}$ Bilen Akuzum, ${ }^{a}$ and E. C. Kumbur ${ }^{a, z}$ \\ ${ }^{a}$ Electrochemical Energy Systems Laboratory, Department of Mechanical Engineering and Mechanics, \\ Drexel University, Philadelphia, Pennsylvania 19104, USA \\ ${ }^{b}$ Laboratoire d'Electrochimie Physique et Analytique, Ecole Polytechnique Federale de Lausanne - Valais Wallis, \\ CH-1951 Sion, Switzerland \\ ${ }^{c}$ Electrochemical Energy Systems and Transport Laboratory, Department of Mechanical Engineering, \\ University of Massachusetts Lowell, Lowell, Massachusetts 01854, USA
}

\begin{abstract}
In this study, we investigate the mass transport effects of various flow field designs paired with raw and laser perforated carbon paper electrodes in redox flow batteries (RFBs). Previously, we observed significant increases in peak power density and limiting current density when perforated electrodes were used in conjunction with the serpentine flow field. In this work, we expand on our earlier findings by investigating various flow field designs (e.g., serpentine, parallel, interdigitated, and spiral), and continuously measuring pressure drop in each configuration. In all cases, these perforated electrodes are found to be associated with a reduction in pressure drop from $4 \%$ to $18 \%$. Flow field designs with a continuous path from inlet to outlet (i.e., serpentine, parallel, spiral) are observed to exhibit improved performance (up to $31 \%$ ) when paired with perforated electrodes, as a result of more facile reactant delivery and resulting greater utilization of the available surface area. Conversely, flow fields with discontinuous paths which force electrolyte to travel through the electrode (e.g. interdigitated), are adversely affected by the creation of perforations due to the high permeability 'channels' in the electrode. These results demonstrate that mass transport can significantly limit the performance of RFBs with carbon paper electrodes.
\end{abstract}

@ 2015 The Electrochemical Society. [DOI: 10.1149/2.0231601jes] All rights reserved.

Manuscript submitted August 5, 2015; revised manuscript received October 26, 2015. Published November 17, 2015. This was Paper 606 presented at the Cancun, Mexico, Meeting of the Society, October 5-9, 2014. This paper is part of the JES Focus Issue on Redox Flow Batteries-Reversible Fuel Cells.

Redox flow batteries (RFBs) are widely considered to be a promising technology for grid-scale electrical energy storage, in applications such as buffering renewable energy sources and time-shifting energy from periods of high supply to periods of high demand (known as peak shaving). ${ }^{1-5}$ The unique aspect of RFBs, as compared with conventional secondary batteries, is that energy capacity and power output are decoupled from each other, such that the energy capacity depends on the volume of the electrolyte storage tanks and the concentration of active species in solution, while the power capacity depends on the total cell area and the number of cells in a stack. Although numerous redox chemistries have been proposed and studied, the all-vanadium chemistry is perhaps the most thoroughly characterized. ${ }^{6-8}$

Regardless of the redox chemistry, the costs associated with these flow battery systems should be minimized to enable their widespread implementation. Previously, it has been shown that the costs associated with the cell stack constitute a substantial portion of the overall system cost. ${ }^{9}$ This has prompted numerous efforts to increase the power density, thereby decreasing the stack size needed for a given power output, and thus lowering the cost of the stack. ${ }^{10-24}$ Previous works have primarily focused on cell geometry, ${ }^{19-24}$ electrode materials and functionalization, ${ }^{10-24}$ and membrane selection. ${ }^{20}$

In 2012, significant improvements in power density were demonstrated by Mench and co-workers by utilizing carbon paper electrodes instead of more conventional carbon felt electrodes. ${ }^{19-22}$ Carbon papers tend to be much thinner than conventional carbon felt electrodes, and thus are less resistive. Moreover, carbon paper is generally denser than carbon felt, resulting in a higher specific surface area and thus more reaction sites. Electrolyte is delivered to the electrode via a flow field which is machined into the bipolar plate, as in fuel cells (a configuration known as 'flow-by', or 'zero gap'). This is in contrast to systems utilizing carbon felt electrodes, where a manifold directs the flow of electrolyte through the plane of the electrode ('flow-through' configuration). Due to the lower hydraulic conductivity of carbon paper versus carbon felt, the flow-by configuration is necessary to have reasonable pressure drop across the cell. However, carbon felt electrodes may also be used in a flow-by configuration. ${ }^{25}$

In a previous work, we hypothesized that the lower hydraulic conductivity of carbon paper electrodes coupled with the flow-by cell configuration could give rise to mass transport limitations, particularly through the thickness of the electrode. ${ }^{24}$ As a means of testing this hypothesis, we created through-plane perforations in the carbon paper electrodes using a laser manufacturing process (Fig. 1a). These electrodes were systematically tested in a 'flow-by' cell configuration with a serpentine flow field to determine their impact on peak power density and limiting current density. A parametric study was performed to determine the optimal perforation diameter and center-to-center spacing. We observed that these perforated carbon paper electrodes offer significant benefits in terms of limiting current density and peak power density. Peak power density was increased up to $30 \%$, while the limiting current density was increased up to $9 \%$, versus unperforated electrodes. These performance improvements were observed despite an overall loss of surface area due to the perforation of the material, and thus were attributed to an enhancement in mass transport within the cell. Moreover, through this process a semi-optimal perforation pattern was identified. ${ }^{24}$

In this work, we seek to expand upon our previous work by combining perforated carbon paper electrodes with several different flow field designs (Fig. 1b) to better understand the mass transport limitations in RFB systems utilizing a 'flow-by' cell configuration. In addition to characterizing the electrochemical performance of each configuration, we have expanded our experimental approach to include measurements of pressure drop across the cell in order to provide more direct evidence of mass transport changes within the cell, and help to identify low pressure drop cell configurations which may minimize pumping power requirements. A detailed analysis and comparison of the system performance for different flow field designs was performed and the results are presented.

\section{Method of Approach}

*Electrochemical Society Active Member.

${ }^{\mathrm{z} E}$-mail: eck32@drexel.edu
Perforation of carbon paper electrodes. - Perforated electrodes were fabricated in-house utilizing the laser-perforation approach 
a)

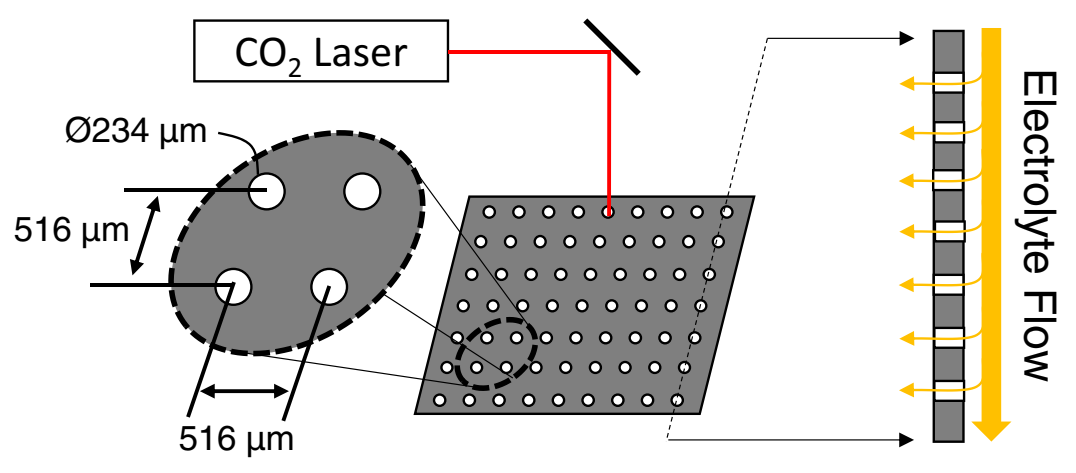

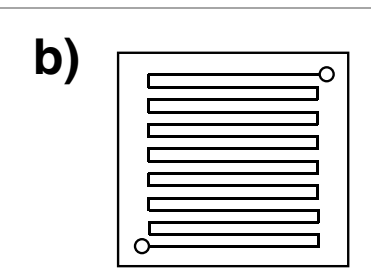

Serpentine

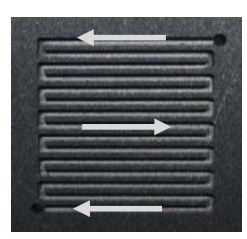

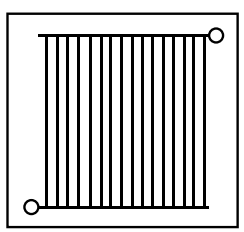

Parallel

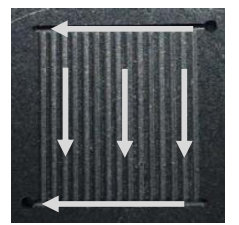

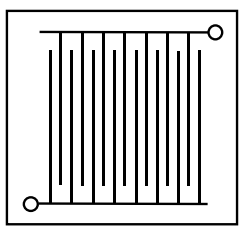

Interdigitated

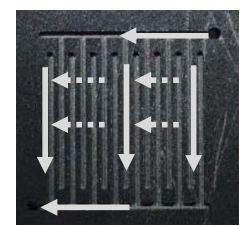

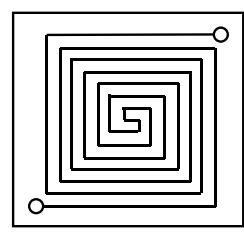

Spiral

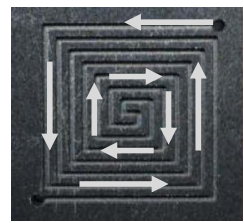

Figure 1. a) Schematic representation of perforated carbon paper electrodes. The perforations act to transport electrolyte from the flow field throughout the thickness of the carbon paper. b) Schematic representations, and photographs of the four flow field designs tested in this work. The solid arrows indicate the direction of electrolyte flow within the flow field, while the dashed arrows indicate flow underneath the ribs of the flow field, through the plane of the electrode.

outlined in Ref. 24. In brief, SGL 10AA carbon paper was affixed to an aluminum plate to ensure a flatness and consistent focusing of the laser. This assembly was placed into a $40 \mathrm{~W}$ laser cutter/engraver (Full Spectrum Laser), and several $5 \mathrm{~cm}^{2}$ electrodes containing an evenly spaced Cartesian grid ( $516 \mu \mathrm{m}$ center-to-center distance, 352.8 holes per $\mathrm{cm}^{2}$ ) of perforations ( $234 \mu \mathrm{m}$ diameter) were manufactured (Fig. 1a). This specific perforation pattern was selected because it demonstrated the best performance in our previous study. ${ }^{24}$ Further characterization of the perforation morphology was previously reported. ${ }^{24}$ These perforated electrodes were then used as-is in the flow cell experiments, without any chemical or thermal treatment.

Design and manufacturing of current collectors.- Current collectors with four different flow field designs (Fig. 1b) were machined from resin-filled graphite plates (GR-940, GraphiteStore.com) using a computer controlled vertical micro-milling machine (Micro Mill DSLS 3000, MicroProto Systems). Serpentine, parallel, interdigitated, and spiral flow field patterns ${ }^{26}$ were designed with a channel width of $0.9 \mathrm{~mm}$ and a channel depth of $1 \mathrm{~mm}$. The serpentine pattern contained 15 evenly-spaced channels in series, while the parallel pattern contained 15 channels in parallel. The interdigitated pattern contained two unconnected flow paths, each with 7 to 8 interlaced 'fingers'. Finally, the spiral flow path spirals toward the center of the electrode 4 times, before spiraling away from the center 4 times. The pattern is such that adjacent channels contain flow in opposite directions. The total electrode contact area for each flow field design is summarized in Table I.

Flow cell.- A custom-designed RFB cell was utilized in this work. Current collectors with different flow field patterns (discussed in previous section) were used to distribute electrolyte within each half-cell, and to provide electrical contact with the electrodes. In all cases, identical flow field designs were used on both halves of the cell. A stack of three layers of SGL 10AA carbon paper was employed as the electrode in each half-cell. Raw (unperforated) SGL 10AA was utilized as a baseline, and compared against the perforated SGL 10AA described previously. Sealing and compression of the electrodes was controlled by utilizing $0.8 \mathrm{~mm}$ thick Viton gaskets, compressing each electrode $\sim 35 \%$. Nafion 117 (DuPont) was soaked in water, and installed between the two compartments to prevent mixing of the electrolyte streams while providing ionic conductivity. Electrolyte flow to/from each half of the cell was accomplished utilizing a high-density polyethylene flow plate, and aluminum end plates were installed on each half to provide even compression and structural support.

Electrolyte preparation. - Vanadium electrolyte at the appropriate oxidation states was prepared via electrolysis, as described in Ref. 10. First, vanadium (IV) oxide sulfate hydrate $\left(\mathrm{VOSO}_{4} \cdot x \mathrm{H}_{2} \mathrm{O}\right.$, Sigma Aldrich) was dissolved into a $4 \mathrm{M}$ solution of sulfuric acid and deionized water to achieve a final vanadium concentration of $1 \mathrm{M}$ in the V(IV) oxidation state. Electrolysis was performed on equal volumes of this starting solution utilizing the flow cell described previously. Following this first electrolysis, oxidation states of V(III) and V(V) were achieved in the negative and positive reservoirs, respectively.

Table I. Total electrode contact area for each flow field design.

\begin{tabular}{cc} 
Flow Field Design & Contact Area $\left(\mathrm{cm}^{2}\right)$ \\
\hline Serpentine & 2.21 \\
Parallel & 2.31 \\
Spiral & 2.46 \\
Interdigitated & 2.51
\end{tabular}


The V(V) solution was then replaced with an equal volume of V(IV) solution, and the electrolysis was repeated again. At the conclusion of this electrolysis, oxidation states of $\mathrm{V}$ (II) and V(V) were achieved in the negative and positive reservoirs, respectively. This configuration corresponds to the fully charged ( $\sim 100 \%$ state-of-charge) state of the battery, containing $\sim 1 \mathrm{M} \mathrm{V}(\mathrm{V})$ and $\sim 0 \mathrm{M} \mathrm{V}(\mathrm{IV})$ on the positive half, and $\sim 1 \mathrm{M} \mathrm{V}$ (II) and $\sim 0 \mathrm{M} \mathrm{V}$ (III) on the negative half.

Electrochemical measurements.-All measurements were performed using a Scribner Associates 857 Redox Flow Cell Test System. The flow cell was fitted with pressure sensors (Dwyer Instruments, 0 50 psi range) at each inlet in order to measure the pressure drop across each half-cell. The pressure sensors were protected from corrosion by fluoropolymer diaphragm 'gauge guards', and hydraulic oil was used to transfer pressure from the diaphragm to the pressure sensor. The analog output from the pressure sensors was integrated with the testing setup using the Scribner 890 Data Acquisition system.

Polarization characterization was performed by applying a series of galvanostatic discharge steps (each $20 \mathrm{~mA} / \mathrm{cm}^{2}$ for $30 \mathrm{~s}$ ), beginning from $\sim 100 \%$ state-of-charge (SOC). Throughout the polarization experiment, the high frequency resistance (HFR) of the system was continuously measured at a frequency of $10 \mathrm{kHz}$, and the pressure drop in each half of the battery was recorded. The polarization experiment was terminated when the cell potential decreased below $0.2 \mathrm{~V}$. At the conclusion of each experiment, the cell was returned to $\sim 100 \%$ SOC by charging at a constant potential of $1.8 \mathrm{~V}$ until the current density dropped below $2 \mathrm{~mA} / \mathrm{cm}^{2}$. The HFR measurements were subsequently used to iR-correct the polarization data. The nominal flow rate for these experiments was $50 \mathrm{~mL} / \mathrm{min}$, which corresponds to an ideal stoichiometric current density of $16 \mathrm{~A} / \mathrm{cm}^{2}$. For the serpentine and interdigitated flow fields, the flow rate was also varied from 2 $\mathrm{mL} / \mathrm{min}$ to $80 \mathrm{~mL} / \mathrm{min}$, corresponding to ideal stoichiometric current densities of $0.64 \mathrm{~A} / \mathrm{cm}^{2}$ and $25.7 \mathrm{~A} / \mathrm{cm}^{2}$, respectively.

\section{Results and Discussion}

Perforated electrodes in a serpentine flow configuration. - In this work, a total of 8 different cell configurations were investigated: 4 different flow fields (serpentine, parallel, interdigitated, and spiral), each coupled with both perforated and non-perforated (raw) carbon paper electrodes. The serpentine flow field is perhaps the most widely used flow field in 'flow-by' RFB cell configurations, and is presented in this section as a baseline configuration. The results of discharge polarization testing at different flow rates using the serpentine flow field configuration are presented in Fig. 2 for both raw and perforated electrodes. As the flow rate is increased, the downward deflection associated with concentration polarization is shifted to larger current densities. This enhancement is expected: as the flow rate increases, the electrolyte residence time within the cell is reduced, leading to a higher average SOC within the cell. Presumably, the electrolyte is also more effectively delivered to less accessible regions of the electrode. However, the improvement becomes marginal beyond 50 $\mathrm{ml} / \mathrm{min}$, suggesting an intrinsic mass transport limitation associated with the electrodes. During the experiments, it was noted that the HFR values measured in this work were larger than our previous study. ${ }^{24}$ This is most likely due to the difference in current collector material. In our previous work, a pyrolitically sealed graphite current collector was used, while in this work relatively less conductive resin-filled graphite was chosen to allow the flow fields to be machined without the need for subsequent pyrolytic sealing of the surface. Additionally, variations in membrane pretreatment and manufacturing, and electrode conductivity may also cause the observed change in HFR.

Figure 3 shows a comparison of the peak power density and limiting current density as a function of flow rate for each electrode type. The relative enhancement achieved by utilizing perforated electrodes is also plotted. In terms of peak power density, perforated electrodes offer between $8 \%$ and $30 \%$ improvement versus the raw electrodes. The greatest enhancement was achieved at a flow rate of $5 \mathrm{ml} / \mathrm{min}$. Below this flow rate, the cell is likely to be starved for reactants, leading to a low peak power density in both cases. The perforated electrodes also enhance the limiting current density between $3 \%$ and $20 \%$.

It is clear that perforated electrodes offer significant performance enhancements when coupled with a serpentine flow field, particularly at lower flow rates. These results closely agree with our previous study of perforated electrodes. ${ }^{24}$ In the following section, we expand our analysis to investigate the coupling between perforated electrodes and other flow field designs.

Perforated electrodes in various flow configurations.- Figure 4a shows the polarization data for the raw electrodes used in conjunction with serpentine, parallel, interdigitated, and spiral flow field configurations at a flow rate of $50 \mathrm{~mL} / \mathrm{min}$. Among these cell configurations, the serpentine flow field provided the highest peak power density $\left(460 \mathrm{~mW} / \mathrm{cm}^{2}\right)$ and limiting current density $\left(623 \mathrm{~mA} / \mathrm{cm}^{2}\right)$, followed by the interdigitated flow field which exhibited $346 \mathrm{~mW} / \mathrm{cm}^{2}$ and 523 $\mathrm{mA} / \mathrm{cm}^{2}$, respectively. The spiral flow field exhibited modest performance, with a peak power density of $222 \mathrm{~mW} / \mathrm{cm}^{2}$ and a limiting current density of $483 \mathrm{~mA} / \mathrm{cm}^{2}$. The parallel flow field, however, exhibits an onset of mass transport losses at relatively low current densities (ca. $175 \mathrm{~mA} / \mathrm{cm}^{2}$ ), severely limiting the peak power and limiting current densities.

For comparison, the same flow field designs were also tested with perforated electrodes (Fig. 4b). When compared with the raw electrodes, it is observed that perforated electrodes provide enhanced
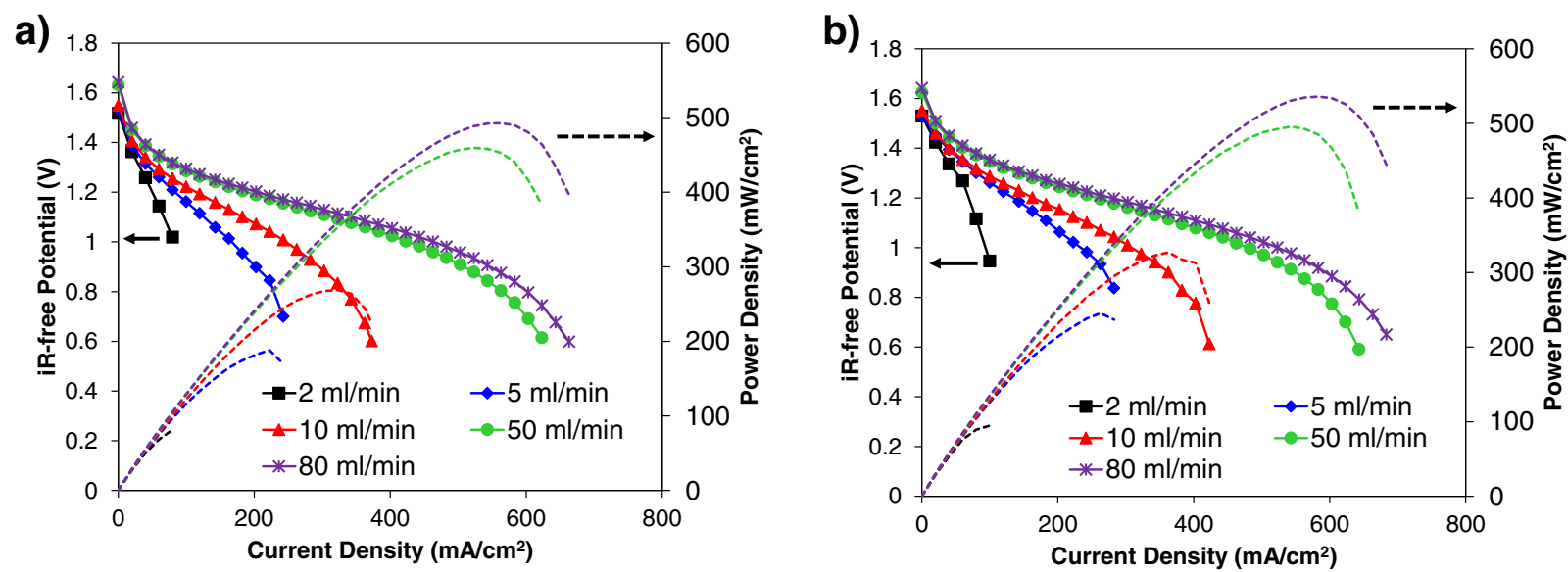

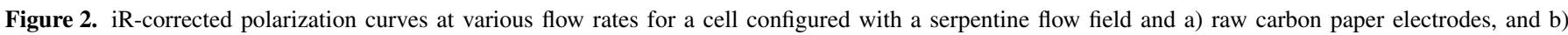
perforated carbon paper electrodes. 
a)

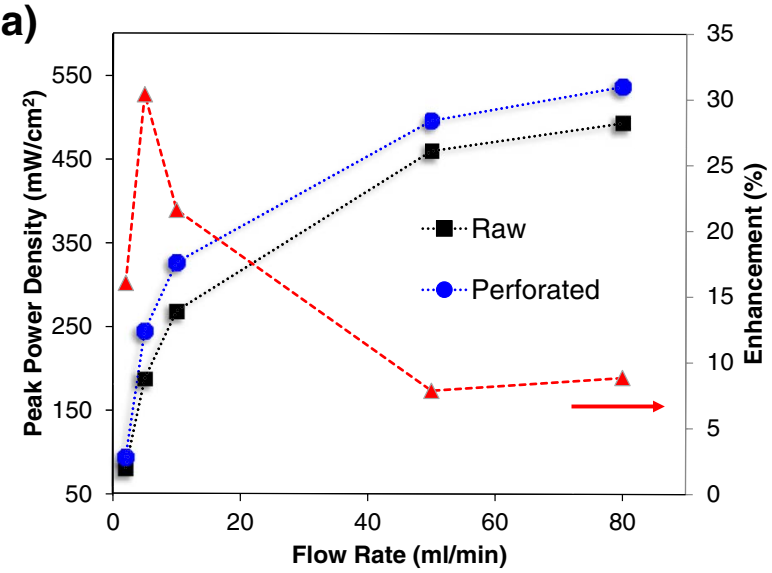

b)

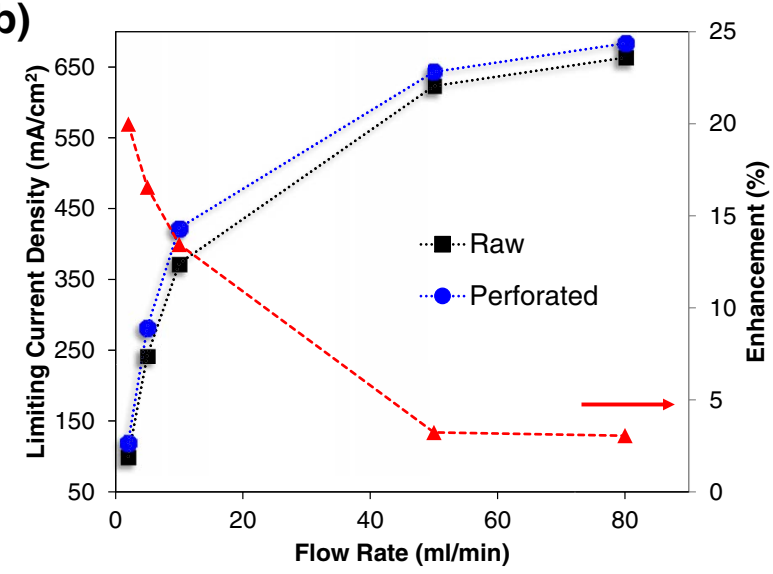

Figure 3. Comparison of a) peak power density and b) limiting current density for the serpentine flow field paired with raw and perforated electrodes. The relative enhancement obtained by utilizing perforated electrodes versus raw electrodes is indicated by (-- $\mathbf{\Delta - - )}$.
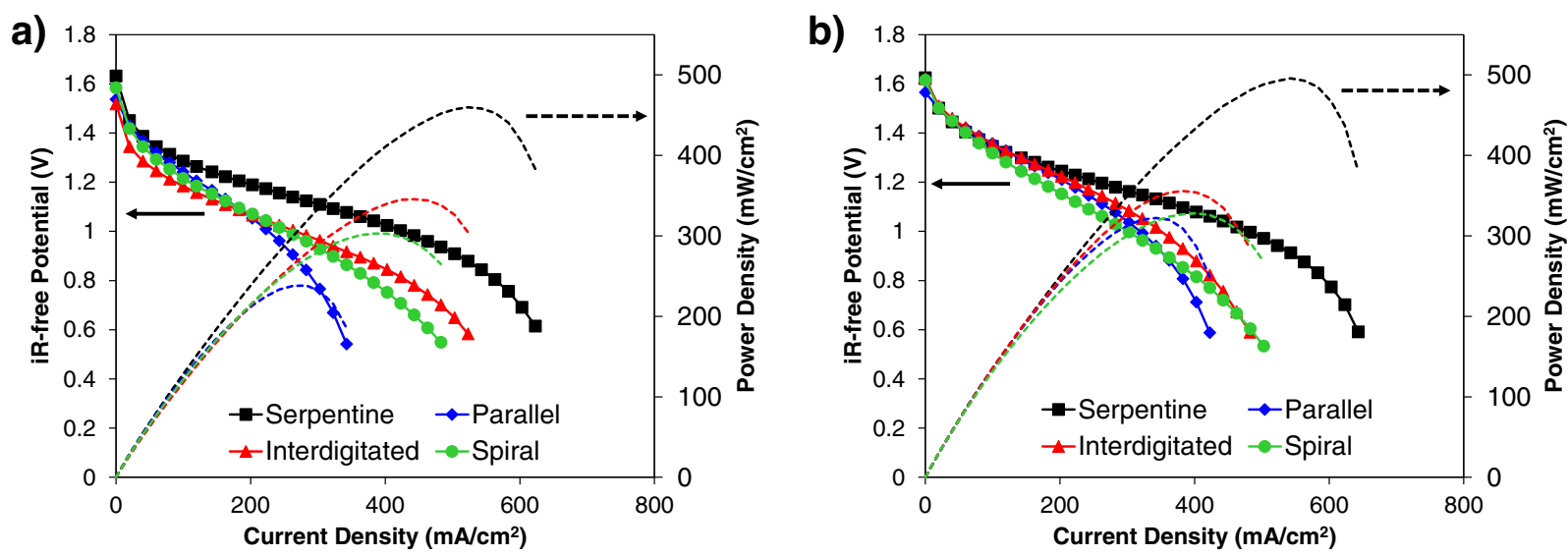

Figure 4. iR-corrected polarization curves at $50 \mathrm{ml} / \mathrm{min}$ for various flow field designs and a) raw carbon paper electrodes, and b) perforated carbon paper electrodes.

peak power density (from $3 \%$ to $35 \%$ ) for all flow field configurations (Fig. 5a). The serpentine flow field exhibited the highest peak power density for both types of electrodes. Although the parallel flow field demonstrated significant mass transport limitations when paired with raw electrodes, the incorporation of perforated electrodes appears to alleviate these losses somewhat, increasing peak power density by $94 \mathrm{~mW} / \mathrm{cm}^{2}$.
However, a comparison of the limiting current densities (Fig. 5b) suggests important differences in mass transport between these configurations. Once again, the serpentine flow field shows the largest limiting current density in both electrode configurations with a slight enhancement by using perforated electrodes. This suggests an improvement of mass transport resulting from the perforations in the electrode. Meanwhile, the interdigitated flow field configuration is
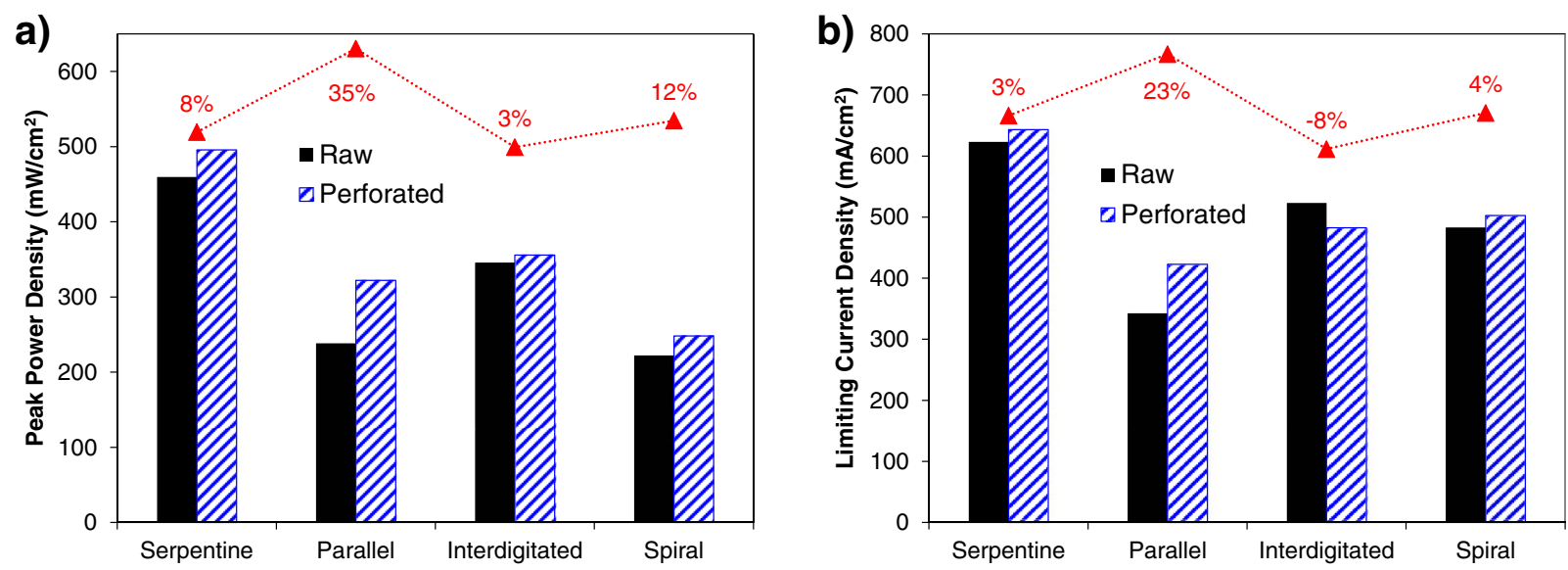

Figure 5. Comparison of a) peak power density and b) limiting current density for various flow fields paired with both raw and perforated electrodes. The relative

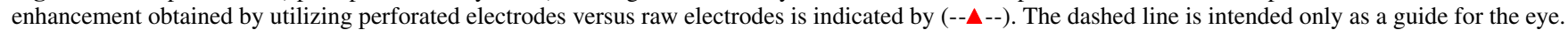



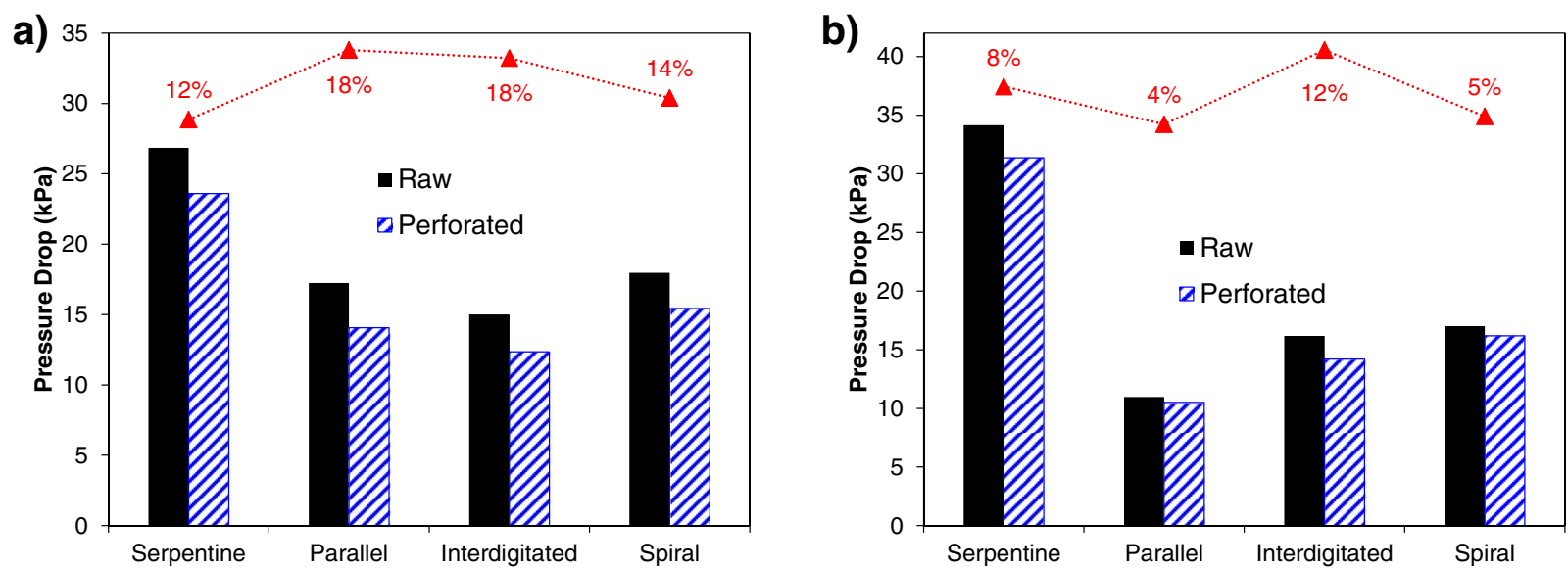

Figure 6. Comparison of pressure drop across the a) positive and b) negative half-cells for various flow fields paired with both raw and perforated electrodes. The relative enhancement obtained by utilizing perforated electrodes versus raw electrodes is indicated by (-- -- ). The dashed line is intended only as a guide for the eye.

not enhanced by the utilization of perforated electrodes, suggesting the perforated electrodes have an adverse effect on the mass transport within this cell configuration. The spiral flow field outperforms the parallel flow field for both electrodes tested, and exhibits a slight enhancement in power density for the case of perforated electrodes. Nonetheless, the largest relative enhancement was observed for the parallel flow field, where the limiting current density was increased $23 \%$ from $343 \mathrm{~mA} / \mathrm{cm}^{2}$ for the raw electrode to $423 \mathrm{~mA} / \mathrm{cm}^{2}$ for the perforated electrode.

Hydraulic effects. - In this work, we have expanded our investigation of perforated electrodes to include measurements of pressure drop in each half-cell. These measurements provide additional insight into the mass transport occurring inside the cell. The pressure drop in each half-cell is presented in Fig. 6, for each flow field and electrode configuration. The pressure drop was measured during the polarization testing, and was not observed to vary significantly during the test. Accordingly, the values shown are the time-average of the data collected during the polarization testing. Moreover, the values represent the total pressure drop from the inlet of the cell to the outlet, and thus include the contribution of the cell fittings and flow manifold, in addition to the pressure drop due to the flow field and the electrodes. These additional contributions represent a constant offset pressure drop for each half cell, but are not necessarily equal between the two half cells. Additionally, the viscosity of the two electrolytes is known to be different, ${ }^{8}$ so under constant flow conditions, a pressure differential between the two half-cells is expected. For these reasons, it is only appropriate to compare values measured on the same half-cell.

For the positive half-cell (Fig. 6a), the interdigitated flow field showed the lowest pressure drop among the flow fields tested. This result is counter-intuitive, as the parallel flow field theoretically offers the shortest, least obstructed flow path for the electrolyte. This may be an experimental artifact resulting from the relatively small flow cell used in this work. The parallel and spiral flow fields each had similar pressure drop, and the serpentine flow field showed the highest pressure drop. The serpentine flow field was also associated with the highest pressure drop on the negative half-cell as well (Fig. 6b). However, the parallel flow field exhibited the lowest pressure drop on the negative half-cell, probably due to the lower viscosity of the negative electrolyte. ${ }^{8}$ For both half-cells, the use of perforated electrodes was associated with an appreciable decrease in pressure drop. This reduction in pressure drop varied from $4 \%$ to $18 \%$, depending on the cell configuration.

The pressure drop was also measured at different flow rates for the two best performing flow field designs (Fig. 7). For the serpentine flow field, utilization of perforated electrodes was associated with a clear reduction in pressure drop, particularly at higher flow rates. Similar behavior was observed for the interdigitated flow field as well, although the effect is less pronounced. At very low flow rates, it appears that the pressure drop is larger for the cases utilizing perforated electrodes. However, the pressure drop measured at these flow rates
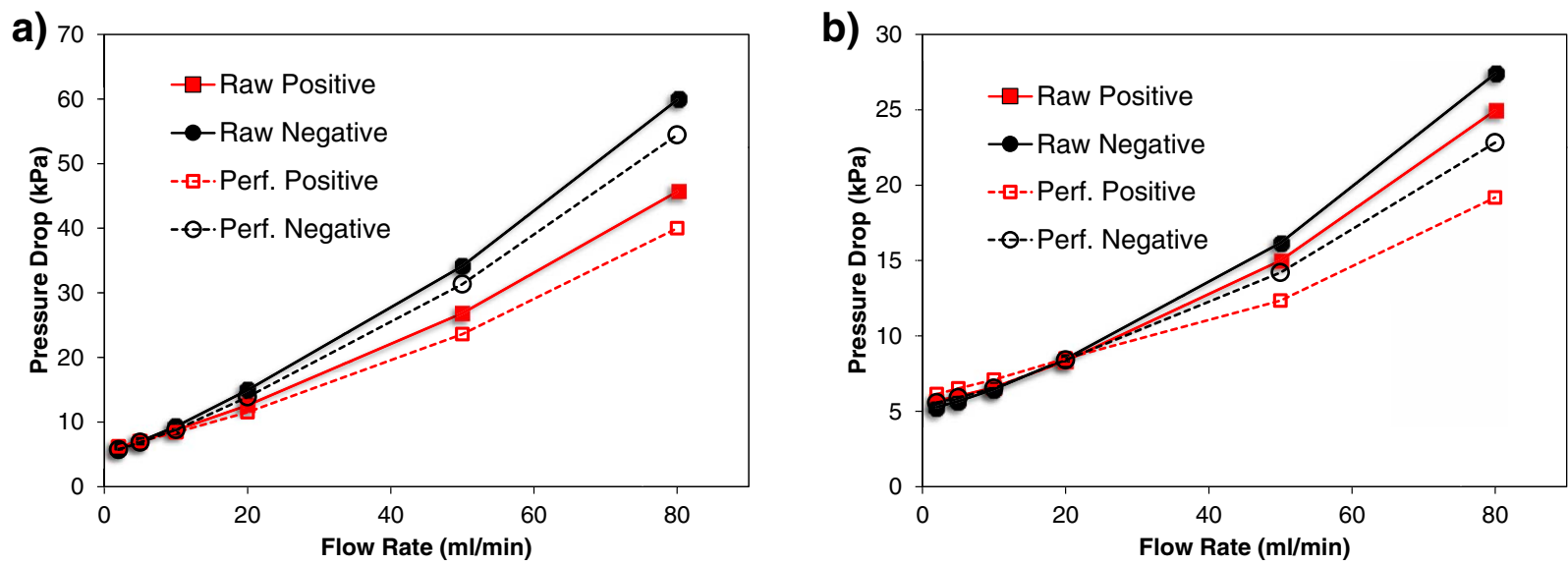

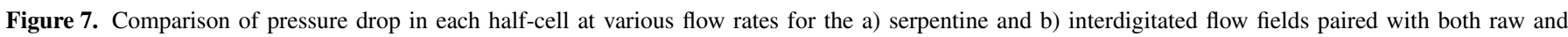
perforated electrodes. 
is quite small, and approaches the sensitivity limit for the pressure sensors used in this study. Thus, these variations are attributed to instrument error.

Discussion on flow field designs. - In this work, the coupling between perforated electrodes and flow field design was investigated experimentally. First we consider the effect of the flow field design without reference to the electrode type. In this work, the serpentine flow field consistently exhibited the highest electrochemical performance. However, the pressure drop associated with the serpentine flow field was also the largest among the flow fields tested. This is due the very long, single flow path. As a result, a large differential pressure can exist between neighboring segments of the serpentine. This creates a tendency for electrolyte to pass ('skip') under the ribs of the flow field, moving in the direction of maximum pressure drop. As a result, some portion of the electrolyte follows the designated serpentine flow path, and the remaining portion travels through the electrode, enhancing electrolyte delivery. However, it is important to note that the test cell used in this work was relatively small $\left(5 \mathrm{~cm}^{2}\right)$. At larger scales, the pumping power required by such a flow field design may become unacceptable. In such cases, a parallel-serpentine (several serpentine flow paths in parallel), or an interdigitated flow field may be a more tenable design choice.

The interdigitated flow field, in particular, appears to offer a reasonable balance between electrochemical performance and pressure drop. The interdigitated design forces the electrolyte to move through the plane of the electrode and thus improves the delivery of the electrolyte. Moreover, although the electrolyte must travel through the relatively dense electrode, the total path length through the cell is much shorter than the serpentine flow field, leading to lower overall pressure drop. This conclusion echoes that of Darling and Perry. ${ }^{25}$

The parallel flow field generally exhibited the lowest electrochemical performance among the flow fields tested. However, the pressure drop associated with the parallel design was generally the lowest as well. This is attributed to the numerous flow paths available as electrolyte passes through the cell. As a result, the flow rate and velocity in each channel is relatively low, and adjacent channels are at similar pressures. Thus, electrolyte flow in the flow field is quite facile, and there is little driving force available for electrolyte transport within the electrode.

The spiral flow field performed surprisingly well in our tests, although it is not generally used for flow battery applications. In general, it performed only slightly worse than the interdigitated flow field, in terms of both electrochemical performance and pressure drop. Indeed, the electrolyte transport in the spiral flow field is quite interesting, and may explain its surprising performance. The flow field consists of two interlaced spirals. As electrolyte enters the cell, it 'spirals' toward the center of the electrode area. Upon reaching the center point, the spiral changes directions and 'spirals' out from the center point, in between the first set of spiral channels (Fig. 1b). Thus, if we assume that electrolyte enters the cell at a high SOC and exits the cell at a low SOC, then each adjacent channel alternates between relatively fresh electrolyte and relatively depleted electrolyte. In effect, this results in a relatively constant areal SOC distribution across the surface of the electrode, with very little gradient from the inlet to the outlet. This should create a relatively uniform current density distribution as well. Additionally, due to the relatively long, interlaced flow path, there can be a large pressure differential between adjacent channels resulting electrolyte skipping under the ribs of the flow field, similar to the serpentine flow field.

This work also reveals the impact of using perforated electrodes in tandem with each of these flow fields. The perforations created in this work were quite large (234 $\mu \mathrm{m}$ diameter) and highly oriented in the through plane direction, improving the hydraulic conductivity. Through-plane mass transport is critical to ensure that electrolyte is effectively delivered throughout the entire thickness of the electrode. For the parallel flow field, mass transport in the electrode is expected to occur primarily in the through-plane direction due to the relatively low pressure drop, and correspondingly low velocities in the flow field. Thus, the significant enhancement in peak power density and limiting current density observed in Figs. 5a and 5b, respectively, can largely be attributed to the improved through-plane hydraulic conductivity of the perforated electrodes.

Additionally, the close spacing of these perforations $(516 \mu \mathrm{m}$ center-to-center distance) is believed to enhance the in-plane hydraulic conductivity of the electrode. In-plane transport is especially important for the serpentine, interdigitated, and spiral flow fields, where electrolyte may 'skip' under the ribs of the flow field in order to enter an adjacent flow channel at a lower pressure. The reduction in pressure drop for each of these flow fields (Fig. 6) can largely be attributed to this enhancement in hydraulic conductivity. Moreover, it suggests greater through-plane electrolyte transport for the perforated electrodes. As a result, the peak power density (Fig. 5a) is enhanced for all flow field configurations. The limiting current density is also consistently increased by utilizing the perforated electrodes, with the notable exception of the configuration using the interdigitated flow field. For this flow field design, an $8 \%$ decrease in limiting current density was observed. This is a rather significant loss, and warrants further consideration.

Among the flow fields tested, the interdigitated flow field is unique because it does not have a continuous path from inlet to outlet, and thus forces electrolyte to travel through the electrode. Although similar behavior ('skipping') may occur in the serpentine and spiral flow fields, it is not mandatory and will not occur in the case of low hydraulic conductivity electrodes. For these flow fields, the perforated electrodes
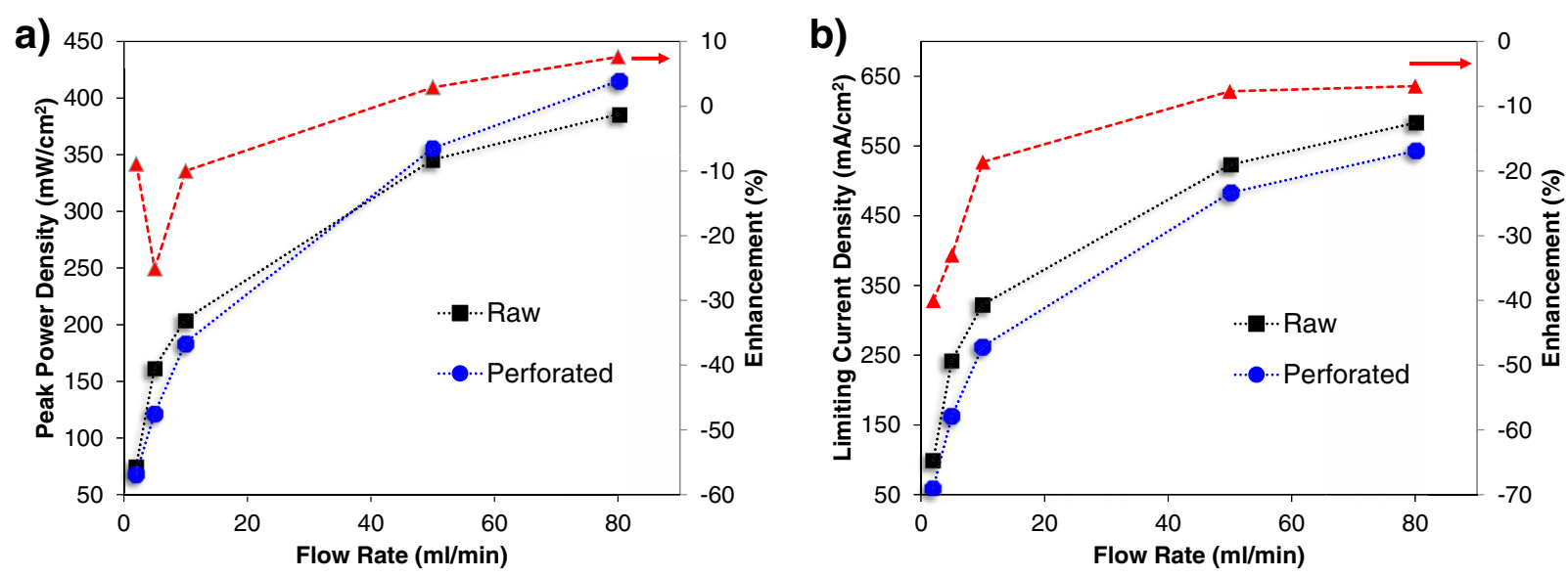

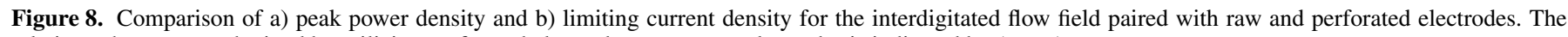
relative enhancement obtained by utilizing perforated electrodes versus raw electrodes is indicated by (-- $\boldsymbol{\Delta}--)$. 
offer higher hydraulic conductivity, and thus encourage this mode of electrolyte transport. The enhancement of limiting current density for these flow field designs suggest that the limiting current is largely dictated by electrolyte delivery. However, for the interdigitated flow field, the electrolyte must 'skip' under the ribs of the flow field, regardless of the hydraulic conductivity of the electrode. Thus, increasing the hydraulic conductivity of the electrode does not enhance this type of behavior; it merely lowers the pressure required (as observed in Fig. 7). For a flow field with excellent electrolyte distribution, such as the interdigitated flow field, the perforations may create high permeability "channels", through which the electrolyte is preferentially transported. These channels may limit mass transport to the available surface area within the electrodes, decreasing the performance of the system.

To further investigate the mass transport using the interdigitated flow field, it is useful to examine the effect of flow rate on electrochemical performance (Fig. 8). As with the other flow field designs, peak power density and limiting current density are observed to increase as the flux of reactants is increased. However, for this flow field, the use of perforated electrodes is consistently associated with a relative decrease in limiting current density versus the raw electrodes. Again, this data suggests that perforations create 'channels' of higher hydraulic conductivity, and the electrolyte is preferentially transported through these channels. As a result, the available surface area in between these channels is poorly utilized.

\section{Conclusions}

In this work, we investigated the effects of various flow field designs paired with raw and perforated carbon paper electrodes on the electrolyte flow and the performance of vanadium redox flow batteries. Overall, the results in this work reinforce the notion that mass transport limitations can significantly hinder the electrochemical performance of RFB cells utilizing carbon paper electrodes. For flow field designs with a continuous path from inlet to outlet (i.e., serpentine, parallel, spiral), creating large diameter, uniformly spaced throughplane perforations in the electrodes is observed to provide substantial improvements in peak power density and limiting current density. Two mechanisms are proposed to explain these improvements. First, the large diameter and highly oriented nature of these perforations provides more facile reactant delivery throughout the thickness of the electrode, enabling greater utilization of the available surface area. Second, these perforations also act to raise the hydraulic conductivity in the through-plane direction. As a result, the electrolyte is more likely to 'skip' underneath the ribs of the flow field, increasing convection in the bulk of the electrode and further enhancing reactant delivery. An additional benefit of utilizing perforated electrodes is the large, consistent decrease (4\% to $18 \%$ ) in pressure drop across the cell, which translates to a decrease in parasitic pumping losses.

Among the tested flow fields, cell configurations based on the interdigitated flow field design did not benefit from the use of perforated electrodes. This suggests that the mass transport in these configura- tions is already sufficient. In fact, the inclusion of perforations may result in mal-distribution of electrolyte, limiting the utilization of the available surface area. Among the cell configurations tested, the interdigitated flow field paired with raw carbon paper electrodes appears to a good balance between pressure drop and electrochemical performance. However, the effects of scaling must always be considered, thus other flow field designs, as well as perforated electrodes, may be more advantageous at larger scales.

\section{Acknowledgments}

Support from the National Science Foundation (grant \#1351161) is acknowledged.

\section{References}

1. M. Skyllas-Kazacos, M. H. Chakrabarti, S. A. Hajimolana, F. S. Mjalli, and M. Saleem, Journal of the Electrochemical Society, 158, R55 (2011).

2. A. Parasuraman, T. M. Lim, C. Menictas, and M. Skyllas-Kazacos, Electrochimica Acta, 101, 27 (2013).

3. T. V. Nguyen and R. F. Savinell, Electrochemical Society Interface, 19, 54 (2010).

4. B. Dunn, H. Kamath, and J.-M. Tarascon, Science, 334, 928 (2011).

5. A. Weber, M. Mench, J. Meyers, P. Ross, J. Gostick, and Q. Liu, J Appl Electrochem, 41, 1137 (2011)

6. E. Sum, M. Rychcik, and M. Skyllas-kazacos, Journal of Power Sources, 16, 85 (1985).

7. E. Sum and M. Skyllas-Kazacos, Journal of Power Sources, 15, 179 (1985).

8. C. Blanc, Modeling of a vanadium redox flow battery electricity storage system, Doctoral Dissertation, EPFL, Thesis No:4277 (2009).

9. M. Zhang, M. Moore, J. S. Watson, T. A. Zawodzinski, and R. M. Counce, Journal of The Electrochemical Society, 159, A1183 (2012).

10. E. Agar, C. R. Dennison, K. W. Knehr, and E. C. Kumbur, Journal of Power Sources, 225, 89 (2013).

11. S. Zhong, C. Padeste, M. Kazacos, and M. Skyllas-Kazacos, Journal of Power Sources, 45, 29 (1993)

12. W. H. Wang and X. D. Wang, Electrochimica Acta, 52, 6755 (2007).

13. Z. Gonzalez, C. Botas, P. Alvarez, S. Roldan, C. Blanco, R. Santamaria, M. Granda, and R. Menendez, Carbon, 50, 828 (2012)

14. B. Sun and M. Skyllas-Kazacos, Electrochimica Acta, 37, 1253 (1992).

15. B. Sun and M. Skyllas-Kazacos, Electrochimica Acta, 37, 2459 (1992).

16. W. Guanjie, J. Chuankun, L. Jianguo, and Y. Chuanwei, Journal of Power Sources, 220, 185 (2012).

17. G. Qiu, C. R. Dennison, K. W. Knehr, E. C. Kumbur, and S. Ying, Journal of Power Sources, 219, 223 (2012)

18. G. Qiu, A. S. Joshi, C. R. Dennison, K. W. Knehr, E. C. Kumbur, and Y. Sun, Electrochimica Acta, 64, 46 (2012).

19. D. S. Aaron, Q. Liu, Z. Tang, G. M. Grim, A. B. Papandrew, A. Turhan, T. A. Zawodzinski, and M. M. Mench, Journal of Power Sources, 206, 450 (2012).

20. Q. H. Liu, G. M. Grim, A. B. Papandrew, A. Turhan, T. A. Zawodzinski, and M. M. Mench, Journal of the Electrochemical Society, 159, 1246 (2012).

21. M. P. Manahan, Q. H. Liu, M. L. Gross, and M. M. Mench, Journal of Power Sources, 222, 498 (2013)

22. A. M. Pezeshki, J. T. Clement, G. M. Veith, T. A. Zawodzinski, and M. M. Mench, Journal of Power Sources, 294, 333 (2015).

23. D. Aaron, C.-N. Sun, M. Bright, A. B. Papandrew, M. M. Mench, and T. A. Zawodzinski, ECS Electrochemistry Letters, 2, A29 (2013).

24. I. Mayrhuber, C. R. Dennison, V. Kalra, and E. C. Kumbur, Journal of Power Sources, 260, 251 (2014).

25. R. M. Darling and M. L. Perry, Journal of The Electrochemical Society, 161, A1381 (2014).

26. M. M. Mench, in Fuel Cell Engines, John Wiley \& Sons, Inc. (2008). 